\title{
Configuration and Dynamic Adaptation of AAL Environments to Personal Requirements and Medical Conditions
}

\author{
Reiner Wichert \\ Fraunhofer Alliance Ambient Assisted Living \\ Fraunhoferstrasse 5, 64283 Darmstadt, Germany \\ reiner.wicherteigd. fraunhofer. de
}

\begin{abstract}
AAL concepts have been shaping scientific and market-oriented research landscapes for many years now [1]. Population development demands have made residing and receiving care in one's own home a better alternative than institutionalized inpatient care. This reality has been reflected in open calls for proposals, as well as in numerous European and domestic projects, and has resulted in a considerable number of applications and product concepts with AAL ties. Unfortunately, it is already foreseeable that these project results will not be implemented in a comprehensive fashion, as individual applications and products can only be combined into a comprehensive solution with a great deal of effort and potential cost. Through stereotypical projects and prototypes, as well as concrete usage scenarios, this paper will extrapolate the added value resulting from integrating individual products into coherent comprehensive solutions within the framework of the complete supply and value chain. Business and technological obstacles will be identified and pathways shown by which AAL concepts and visions can lead to a better reality for all of those concerned, from healthcare recipients to those bearing the costs.
\end{abstract}

Keywords: Ambient Assisted Living, User Interfaces, Elderly People, End User Configuration, AAL Platform.

\section{Increase in the Elderly Living Alone}

In 2005, there were 82.4 million people living in Germany. According to prognoses of the German Statistical Office, this number will decrease to between 68.7 and 79.5 million residents by the year 2050. At the same time, the number of 80 -plus-year-old residents will rise from 4 million (2005) to approximately 10 to 11.7 million (2050) [2]. With increasing age, the proportion of those living alone also increases. In 2000, 44 percent of private households occupied by 65 - to 70 -year-old primary residents were single occupancy dwellings.

In light of increasing divorce rates and the growing number of single, as well as single parent households, this trend is expected to continue [3]. Age in and of itself is not necessarily an indicator for being in need of care, but with increasing age, a higher percentage of the population grows dependent on assistance, support and care giving. 
$96 \%$ of those 70 and older have at least one internal, neurological or orthopedic disease process requiring treatment, whereas $30 \%$ have five or more [4].

Despite the paradigm „out-patient before in-patient“, data from care giving statistics reveal a trend toward professional care. Especially in the area of inpatient care, the number in need of care has risen over the last several years: in 2005, 676,000 people were in nursing home care; in 1999, this number was just 573,000. Current developments, such as the conceptualization and implementation of alternative forms of habitation, take the discussion of an „out-patient conversion“ of care giving under consideration.

The primarily undesired increase in the number of residents in in-patient facilities highlights the current care giving dilemma, namely, that it is very difficult for those in need of outside care and support to continue to live in their accustomed environments. The realization of technologically supported, AAL-based concepts can contribute to closing these care giving loopholes.

\subsection{The Desire for Independent Living in the Golden Years}

The majority of the elderly want to remain in their accustomed environment, even as the need for outside support and care giving increases. Institutionalized forms of habitation, on the other hand, are experiencing a decreasing level of acceptance according to a representative survey by the Schaderstiftung [5]. Independence and selfdetermination have high social value, also among the elderly population. Within the framework of the Fraunhofer IAO project „Pflege 2020” (Care giving 2020), 500 people aged 55-75 were questioned as to their desires and needs for future care giving. Key topics of the representative survey included desired services, forms of habitation, and technological applications. A few guiding universal themes could be identified as fundamental earmarks of a high quality of life according to those surveyed and can be characterized by the keywords "security", "participation", "individuality" and "daily structure".

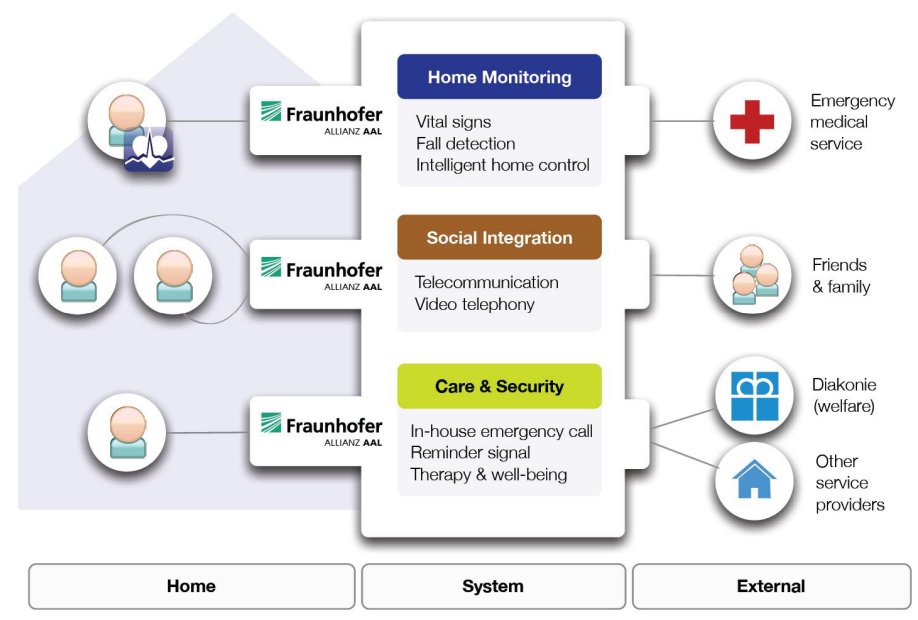

Fig. 1. Application areas for AAL and "Personal Health" concepts in the housing industry 
The effects of the demographic transformation pose enormous challenges for the housing industry, as well. One such challenge is that of keeping older residents with limited mobility and diminishing health in existing housing and to avoid vacancies. Suboptimal housing structures from the 1950s/60s often make a senior-appropriate transformation difficult. Technological support for senior living does not have to be limited to the adaptation of the personal living space, however. It is more pertinent to develop comprehensive concepts that link individual living spaces with a residential quarters-oriented infrastructure. Only the linking of ambient technologies with individualized, health-oriented service concepts can meet the needs and desires of elderly residents and become the foundation for new business models for the housing industry.

\subsection{State of the Art in AAL and Personal Health}

Currently, further development of technological solutions is occurring in a number of predominantly European research ventures. At the beginning of 2007, a total of 16 individual AAL projects were launched within the 6th Framework Program by the European Commission with such diverse thematic focal points as, i.e., social integration, support for daily life, security and mobility (EU-IST PERSONA) [6], semantic service-oriented infrastructure (AMIGO) [7], special support for the blind (HAH Hearing at Home), secure »white goods «(EASY LINE+), Entertainment and Health (OLDES), mobile (cellular) support within the home and elsewhere (ENABLE), support for »Daily Life « and Health Care and Communication (NETCARITY), »Health « vital function monitoring, activities, position (CAALYX, EMERGE), automation between »white goods«, entertainment with variable user interface (IN-HOME), scalable, adaptive, customizable add-ons for personal assistance sensor systems (SHARE-IT), monitoring of daily activities and incorporation of biofeedback (SENSACTION-AAL), and many more. 2008 saw the launch of additional projects from the 7th Framework Program exploring these very topics. Through the continuation of pilot projects, such as "SmarterWohnenNRW" (Smarter Living in Northrine Westphalia) or the application project "Sophia", with complete Internet integration, as well as that of communication and telemedicine, better conditions for the future implementation of AAL solutions in rental housing are being prepared.

For many of these projects, the term "personal health" - like the term AAL - has been playing an increasingly important role. Similar to how the "personal computer" established itself as an extension and complement to professional computer technology, "personal health" denotes the accessibility of devices previously used only by medical personnel - but also that of respective information and service options - now available to the private user.

"Personal health" also characterizes the direction of a paradigm transformation from traditional healthcare to person-centered, individualized prevention, diagnostics, therapy and care giving. This transformational process is supported by developments in the area of telemonitoring, as well as further developments in personalized medicine, which enable the person-oriented integration of digital patient data (images, vital function monitoring, demographic and anamnestic data, lab findings) through the intensive application of information technology and telematics (eHealth), while incorporating the latest developments in biotechnology, genomics, and pharmacology [8], [9]. 
The technology applied in "personal health care" encompasses wearable medical devices or systems in particular, conceptualized for the diagnostic and therapyaccompanying application in the home environment. Such a telemonitoring system typically consists of medical sensors and a base station, either worn by or located in the immediate vicinity of the user. This base station captures data delivered by the sensors, prepares the data if necessary and makes it available via a wired or wireless transmission system to the stationary (AAL) infrastructure, or, if required, to a doctor, hospital or telemedical service provider, where further evaluative steps or data storage can occur.

The sensor devices placed on or in the body communicate with the base station via a wireless network of limited range (Body Area Network / Personal Area Network). The base station can be either a stationary personal computer system with a fixed network connection or a mobile device (Smartphone, PDA, etc.) with wireless transmission technology (GSM, UMTS, WLAN).

In order to develop markets for "personal health" systems and applications - initially based on conditions in the US - an international alliance dubbed "Continua Health Alliance" (www.continuaalliance.org) was founded in 2006 and currently encompasses approximately 170 companies.

A prerequisite to the realization of "personal health" is the availability of reasonably priced, stand-alone, user-manageable system components, as well as their crossmanufacturer interoperability in "open" systems. To enable this availability, Continua guidelines will be compiled as "recipes" for the development of interoperable products supported by a comprehensive system of international norms and industry standards. In addition to fitness and wellness, the intended application areas also encompass chronic disease management outside of clinical environments, as well as support for independent senior living, with the goal of living as long as possible in one's home environment.

\section{Individualized Housing Development and Adaptation}

While the need and market potential for universal AAL applications is clear, there is currently a lack of marketable products that significantly rise above purely isolated applications. Viable, innovative services remain on our wish list, a distant, albeit desirable dream. Some of the isolated applications currently available include home emergency systems, which are constructed as pure alert signallers, sensors for light control, or device-specific user interfaces. These applications could only be linked together with a great deal of effort, with any emergent alterations requiring the involvement and expertise of system specialists, thereby increasing solution costs considerably. In addition, sensors and other hardware components, as well as individual functionalities, have a tendency to require multiple installations with multiple associated costs, as the systems are only offered as complete packages, data exchange formats and protocols are not compatible, and components from one application cannot be used by another application. Likewise, it is impossible to generate higher value functionalities through combining layers of individual functions. In other words, targeted AAL systems are not realizable. 
By contrast, future AAL solutions for the care and support of the elderly must be based on a flexible and expandable platform and be modular and expandable so as to remain adaptable to the individual's changing needs, lifestyle and health.

\subsection{Realizing the Vision through an Integrated Concept}

This scenario presupposes that it will actually be possible to dynamically adapt existing housing to the requirements of age-appropriate living. It is not enough that new components and devices can independently integrate themselves into the current infrastructure. Tools are also required that allow service providers to optimize available resources (services, sensors, device functions) for these infrastructures. With the targeted configuration option, any combination of existing functionalities can be reused in new applications, ultimately leading to the targeted universality, as well as the associated reduction in costs.

The Fraunhofer Alliance AAL, the Fraunhofer frontline theme „Assisted Personal Health", as well as the Fraunhofer innovation cluster "Personal Health", each with expertise in their specific technological fields and in cooperation with external partners, are all contributors in realizing this vision. It is essential to include the complete chain of players in the healthcare field and to get medical professionals, health insurance companies, health associations and organizations, social and health service providers, healthcare lobbyists, housing industry specialists, psychologists, as well as the respective technology developers, all together at one table, in order to develop new forms of cooperation between all participants. It is equally essential to link healthcare assistance in the sense of AAL with personalized information processing, information transfer and information management according to the ,personal health“ paradigm and to further develop these linked components into one comprehensive, universal system.

This integrated process chain approach appears to contradict demands for a quicker realization and marketability as they are raised by the respective industries. The participating institutes want to counter this supposed contradiction by stating that whereas exemplary existing prototypes close to production are first being further developed into marketable products, standard interfaces are being simultaneously prepared for a later integration of existing platforms.

\subsection{Adaptation for Future Needs and Health Issues}

The goal is to equip existing housing with ambient technology such that it can be adapted for future needs and health issues as easily as possible. The focus is on people with chronic illnesses, who can be provided care via telemonitoring. This links to flexible services ensure comprehensive care. Telemedical care can take place, for instance, through a medical service centre and emergency care through a nearby urgent care centre. In addition to the recording of vital functions, the detection of accidental falls is a priority. Participation in societal life for individual residences to the outside world is to be supported by telecommunication. Reminder functions simplify the structuring of the day's activities and simultaneously improve the quality of care and the self-management abilities of the chronically ill. 
An accompanying evaluation would prove useful for purposes of ascertaining which technological components should be linked together to optimize the adaptation of the residences to the individual needs of the residents. In a further step, resident requirements, results and experiences in existing housing should be compiled and fundamental infrastructure prerequisites for future accommodations in housing should be formulated for the housing industry. In summary, the following objectives in particular are essential: (1) Support for independent, autonomous living for the resident in later years and with potential health-related limitations, (2) Enabling a life lead with a high level of security and social living quality, (3) Enhancement of the selfmanagement of chronic diseases and increase in compliance through supportive ambient functions, (4) Expansion of the service portfolio for health-related and social service providers, as well as for the housing industry, (5) Development of an intuitive, operable configuration tool for device adaptation and data access and (6) Development of adaptable technical installations for increased flexibility in view of changing living requirements for existing and new housing.

\section{Solution: Provision of Flexible and Expandable Platforms}

The overriding technological objective is the provision of a flexible and expandable platform for the care and support of the elderly in their home environment. New housing complexes need to be fundamentally constructed such that each residence can be individually adapted to the respective residents. Existing housing is to be retrofitted such that it can be dynamically adapted to the requirements of an aging population if at all possible. It would appear reasonable to follow a two-step approach: In phase one, AAL technologies are to be integrated into existing housing. In phase two, the fundamental infrastructure requirements for the future housing industry are to be developed in new housing construction on the basis of an expandable platform. Further, the integration of sensors worn on the body and medical devices is to be enabled and evaluated incorporating a central basis of information.

Additional components and devices must be fundamentally capable of autonomous integration into these infrastructures [10], [11]. There are validated project results from EU-IST projects, such as PERSONA, AMIGO or SOPRANO, with a special focus on dynamic distributed infrastructures for the self-organization of devices, sensors and services. These results should be taken under consideration [12].

The PERSONA infrastructure e.g. with its four communication buses aims at the provision of mechanisms that facilitate the independent development of components that nonetheless are able to collaborate in a self-organized way as they come together and build up an ensemble. Therefore, the buses act as brokers that resolve the dependencies at runtime using the registration parameters of their members and semantic match-making algorithms [13].

The open nature of such systems must allow dynamic plugability of components distributed over several physical nodes. It consists of a middleware solution for open distributed systems dealing with seamless connectivity and adequate support for interoperability that makes use of ontological technologies and defines appropriate protocols along with an upper ontology for sharing context [14]. 


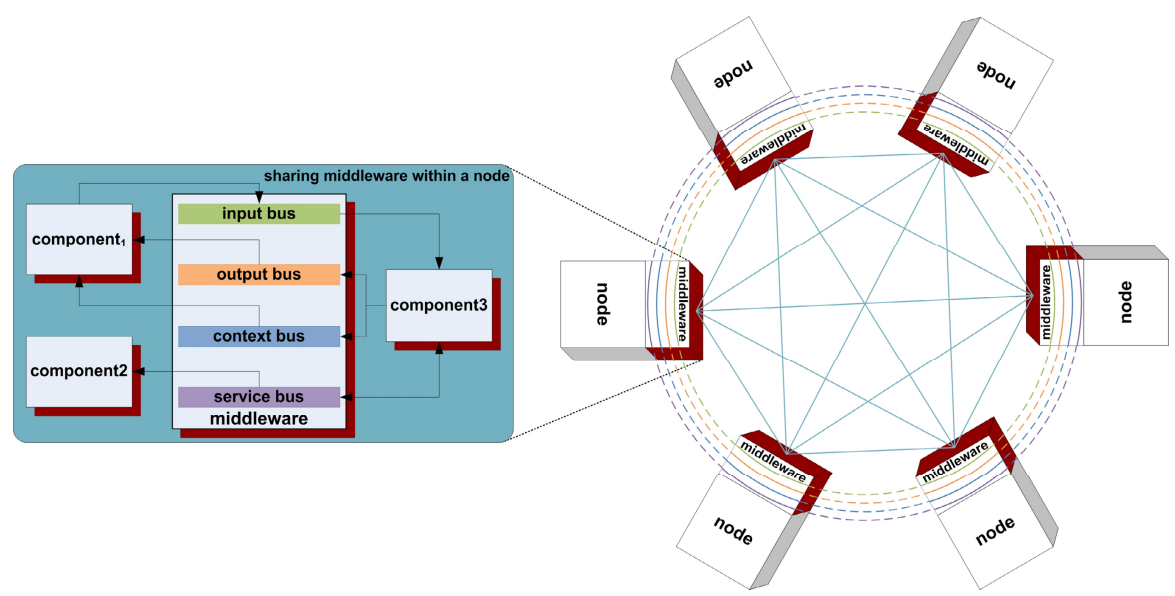

Fig. 2. Decentralized software infrastructure (PERSONA)

\subsection{Configuration Tools - Constraints and Functions}

On the basis of these infrastructures, tools for service providers can be designed, which enable the optimization and configuration of available resources. The targeted configurations should enable higher value functions resulting from a cooperation of resources, thereby generating an added value that has been unattainable up to this point. It is essential that any needs or relevant situations be automatically recognized, analyzed and associated with the call for corresponding functions.

Unfortunately, it is almost never the case that a direct deduction of a reaction is possible on the basis of an event, since (1) situations are not always directly measurable and, therefore, conclusions regarding the situation cannot be based on single events. Rather, it is imperative to draw conclusions based on several events or facts (event aggregation). The situation "resident has fallen", for instance, can be recognized with a greater degree of certainty, if, in addition to an alert sent by one of the acceleration sensors located in the resident's cane that the cane has fallen on the ground, the camera-based analysis of the positioning of a human form ("is in the prone position") is also reported and taken under consideration. It should likewise be taken into account that (2) required functions are not always provided by individual devices and components found in a given environment, but that the desired effect can perhaps only be achieved through the combination of several available functions (composition of services). For instance, a service provider receives an alert about a fall and there is an immediate automated address generated through the resident's environment, asking whether he is alright.

In this scenario, the combination of several functions could prevent false alarms, etc. Unfortunately, associations (links) of situations with the respective functions could change, meaning something that was wanted a particular way up until now could suddenly be interpreted and handled differently (i.e., if a resident's health situation were to suddenly change). 
Configuration tools can also serve to make adaptations to the individual preferences, capabilities and limitations of the resident or for the specific health situation (i.e., with the question as to whether the neighbour, a relative, nursing home personnel, or a combination of the above should be notified of a certain event).. These tools become an essential complement to open systems, which can continue to evolve over longer periods of time. The software infrastructure, for instance, of PERSONA is already in the position to integrate new components ad hoc or to execute aggregate events and services by means of a script.

\subsection{Intuitive Interaction Concepts}

In the (not so distant) future, novel interaction forms will essentially shape everyday life as we know it. Interaction concepts for the control of objects in AAL environments will no longer be centrally realized, as is common, for instance, with the PC. Instead, they will be implemented through networks of (computer) nodes that will interpret user commands and distribute them by way of existing communication infrastructures to the end devices that can best realize the task at hand.

Multimodal interaction concepts, such as speech and gesture recognition or computer vision, require computationally intensive algorithms, which can only be executed by stationary computers. Should additional intelligent deductions from existing information be required, the temporarily increased computational effort can still be generated quickly enough through distributed (computer) nodes. Applications of such interaction concepts include speech interfaces, 3-dimensional interactive video interfaces or emotive interfaces for robots [15].

The potential applications of novel interaction forms can be illustrated by the home environment: In contrast to current concepts with central controls, where functionalities are laboriously programmed and the user must also remember which functions are being activated by which keys, interaction in the AAL environment is decoupled from the hardware. The user no longer uses commands to control devices. Rather, he provides goals that are then interpreted and automatically realized. For instance, if the user acoustically provides the goal ,brighter“, first, the room in which the user currently finds himself will be ascertained. Then, the system will check which options are available for increasing the brightness in this room: Are there blinds which can be opened? What kind of lamps are available? With all actions, the status of the environment is ascertained, as well, as it makes no sense, for instance, to open the blinds at night. The preferences and other goals of the user are also taken into account. So, for watching television, the system could select indirect lighting, but for a work situation or for reading, direct lighting could be chosen.

It is apparent that intelligent environments also require a configuration of the rules, as each user has a preference for his own personal settings and would like to make any modifications himself. In contrast to the approach presented in section 3.2, the users possess less technical know-how in the handling of rules in complex control systems. Conventional menu-based approaches, such as found with mobile telephones, fail due to the sheer number of modification options. Novel interaction forms are, therefore, necessary for configuration of the environment by the residents for acceptance purposes (end-user configuration) and have extensive research potential. 


\section{Conclusion}

The AAL-vision is that one day sensors and systems give seniors a helping hand in their own home by measuring, monitoring and raising alarms if necessary. To reach this goal lot of scientific and market-oriented research have been done in the past years. Unfortunately, people normally have many problems that cannot be solved with a single product. Thus it is already foreseeable that the needed project results will not work together since individual applications and products can only be combined into a comprehensive solution with a great deal of effort. Future AAL applications, however, must be both flexible and expandable, specifically incorporating "personal health" components, in order to be dynamically adaptable to individual demands and respective medical conditions. In contrast, with the current closed system concepts, sensors or functionalities must be potentially installed and paid for multiple times, as the functionality of an application cannot be used by another application. The next step that should be made now would be to get the industry together at a table in order to work out certain standards. Only that way the products would become efficient and also cheaper. Thus if we would not change our strategies in AAL from individual products into coherent comprehensive solutions within the framework of the complete supply and value chain, there will be a huge risk that we have been spend a lot of money in AAL system solutions at the end and AAL would have been only a huge bubble.

\section{References}

1. Emiliani, P.L., Stephanidis, C.: Universal access to ambient intelligence environments: Opportunities and challenges for people with disabilities. IBM Systems Journal 44(3), 605-619 (2005)

2. Federal Statistical Office: Population of Germany till 2050. 11th coordinated population forecast. Wiesbaden, p. 43 (2006)

3. Cirkel, M., et al.: Produkte und Dienstleistung für mehr Lebensqualität im Alter - Expertise. Gelsenkirchen, p. 8 (2004)

4. Robert Koch Institut: Themenheft 10: Gesundheit im Alter, Gesundheitsberichterstattung des Bundes. Berlin (2005a)

5. Heinze, R.G., et al.: Neue Wohnung auch im Alter - Folgerungen aus dem demographischen Wandel für Wohnungspolitik und Wohnungswirtschaft. Schader-Stiftung, Darmstadt (1997)

6. Avatangelou, E., Dommarco, R.F., Klein, M., Müller, S., Nielsen, C.F., Soriano, S., Pilar, M., Schmidt, A., Tazari, M.-R., Wichert, R.: Conjoint PERSONA - SOPRANO Workshop. In: Sala Soriano, M.P., Schmidt, A., Tazari, M.-R., Wichert, R. (eds.) Constructing Ambient Intelligence: AmI 2007 Workshops, pp. 448-464. Springer, Heidelberg (2008)

7. Georgantas, N., Ben Mokhtar, S., Bromberg, Y., Issarny, V., Kalaoja, J., Kantarovitch, J., Gerodolle, A., Mevissen, R.: The Amigo Service Architecture for the Open Networked Home Environment. In: 5th Working IEEE/IFIP Conf. on Software Architecture (WICSA 2005), pp. 295-296 (2005)

8. Blobel, B., Norgall, T.: Standard based Information and Communication - The Personal Health Paradigma. HL7-Mitteilungen, Heft 21/2006, pp. 33-40 (2006) 
9. Norgall, T., Blobel, B., Pharow, P.: Personal Health - The Future Care Paradigm. In: Medical and Care Compunetics 3. Series Studies in Health Technology and Informatics, vol. 121, pp. 299-306. IOS Press, Amsterdam (2006)

10. Aarts, E., Encarnação, J.L.: Into Ambient Intelligence. In: Aarts, E., Encarnaçao, J. (eds.) True Visions: Tales on the Realization of Ambient Intelligence, ch. 1. Springer, Heidelberg (2005)

11. Wichert, R., Tazari, M.-R., Hellenschmidt, M.: Architektonische Requirements for Ambient Intelligence. IT - Information Technology, 13-20 (January 2008)

12. Hellenschmidt, M., Wichert, R.: Rule-Based Modelling of Intelligent Environment Behaviour. In: Künstliche Intelligenz: KI, vol. 2, pp. 24-29 (2007)

13. Furfari, F., Tazari, M.R.: Realizing ambient assisted living spaces with the PERSONA platform. ERCIM News (74), 47-48 (2008)

14. Fides-Valero, Á., Freddi, M., Furfari, F., Tazari, M.-R.: The PERSONA Framework for Supporting Context-Awareness in Open Distributed Systems. In: Aarts, E., Crowley, J.L., de Ruyter, B., Gerhäuser, H., Pflaum, A., Schmidt, J., Wichert, R. (eds.) AmI 2008. LNCS, vol. 5355, pp. 91-108. Springer, Heidelberg (2008)

15. Adam, S., Mukasa, K.S., Breiner, K., Trapp, M.: An Apartment-based Metaphor for Intuitive Interaction with Ambient Assisted Living Applications. In: Proceedings of HCI 2008, Liverpool, May 1-9 (2008) 